\title{
Two Novel Mutations and a de novo Mutation in PSEN1 in Early-onset Alzheimer's Disease
}

\author{
Yu-Sheng Li ${ }^{1, \#, ~ Z h i-H u a ~ Y a n g ~}{ }^{1, \#, ~ Y a o ~ Z h a n g 1, ~ J i n g ~ Y a n g 1, ~ D a n-D a n ~ S h a n g ², ~ S h u-Y u ~ Z h a n g 1, ~}$ \\ Jun Wu', Yan Ji', Lu Zhao ${ }^{1}$, Chang-He Shi ${ }^{1}{ }^{*}$, Yu-Ming $\mathrm{Xu}^{1}{ }^{1 *}$ \\ ${ }^{1}$ Department of Neurology, The First Affiliated Hospital of Zhengzhou University, Zhengzhou University, \\ Zhengzhou, Henan, China. \\ ${ }^{2}$ Department of Neurology, Luoyang Central Hospital affiliated to Zhengzhou University, Henan, China
}

[Received October 2, 2018; Revised November 2, 2018; Accepted November 9, 2018]

\begin{abstract}
Presenilin 1 (PSEN1), presenilin 2 (PSEN2), and amyloid precursor protein (APP) mutations are responsible for autosomal dominant early-onset Alzheimer's disease (AD-EOAD). To analyze the phenotypes and genotypes of EOAD patients, we performed comprehensive clinical assessments as well as mutation screening of PSEN1, PSEN2, and exons 16 and 17 of APP by Sanger sequencing in the three Chinese EOAD families. We identified two novel mutations of PSEN1 (Y256N and H214R) in samples from these families, and a de novo mutation of PSEN1 (G206V) in a patient with very early-onset sporadic Alzheimer's disease. A combination of bioinformatics tools based on evolutionary, structural and computational methods predicted that the mutations were all deleterious. These findings suggest that PSEN1 Y256N, H214R, and G206V need to be considered as potential causative mutations in EOAD patients. Further functional studies are needed to evaluate the roles of these mutations in the pathogenesis of $\mathrm{AD}$.
\end{abstract}

Key words: Early-onset Alzheimer's disease; EOAD, Mutation, PSEN1

Alzheimer's disease (AD) is the most common form of dementia and has a high incidence rate among the elderly people [1]. $\mathrm{AD}$ can be categorized into early-onset Alzheimer's disease (EOAD, <65 years of age) and lateonset $\mathrm{AD}$ (LOAD, $>65$ years of age). Most $\mathrm{AD}$ cases are $\mathrm{LOAD}$, whereas $\mathrm{EOAD}$ is reported quite rarely, accounting for $5-10 \%$ of all the $\mathrm{AD}$ cases [2]. To date, three genes responsible for autosomal-dominant EOAD (AD-EOAD) have been identified: presenilin 1 (PSEN1), presenilin 2 (PSEN2), and amyloid precursor protein $(A P P)[3,4]$. Besides, there are also several de novo EOAD mutations detected in very early-onset sporadic cases of $\mathrm{AD}$ [5-10].

Mutations in PSENI are the most frequent mutations in AD-EOAD patients. The PSEN1 gene (NM_000021.3) contains 10 coding exons and three noncoding exons. It encodes the PSEN1 protein, which consists of nine transmembrane (TM) domains and a hydrophilic loop (HL) region [11]. PSEN1 is the major component of the catalytic subunit of the $\gamma$-secretase complex performing APP cleavage [12]. Mutations in PSEN1 could lead to over-production of amyloid $\beta$ 1-42 and amyloid deposits in the brain [13]. Until now, more than 200 pathogenic mutations in the PSENI gene have been reported. EOAD cases with PSENI mutations usually occur at 40-60 years of age, but several PSEN1 mutations have also been reported to be associated with very early-onset $\mathrm{AD}$ (vEOAD; <35 years of age) [14]. Nonetheless, there are still mutations that remain unidentified along with the rarely reported de novo mutations [15]. In this study, we aimed to present the phenotypic and genetic analysis of three Chinese EOAD families.

*Correspondence should be addressed to: Drs. Yu-ming Xu and Chang-he Shi, The First Affiliated Hospital of Zhengzhou University, Zhengzhou University, Zhengzhou, China. Email: xuyuming@zzu.edu.cn or shichanghe@ gmail.com. \# These authors contributed equally to this work.

Copyright: (C) 2018 Li YS et al. This is an open-access article distributed under the terms of the Creative Commons Attribution License, which permits unrestricted use, distribution, and reproduction in any medium, provided the original author and source are credited. 


\section{MATERIALS AND METHODS}

\section{Subjects}

Three Chinese EOAD families were enrolled in the study. EOAD was diagnosed independently by two neurologists on the basis of the criteria of the National Institute of Neurological and Communicative Disorders, and the Stroke-Alzheimer's Disease and Related Disorder Association (NINCDS-ADRDA) [16]. Each patient was assessed via a series of neuropsychological scales, including the Mini-Mental State Examination (MMSE), Montreal Cognitive Assessment (MoCA), Clinical Dementia Rating (CDR), and Alzheimer's Disease Assessment Scale-cognitive (ADAS-cog). Besides, 200 healthy Chinese volunteers were enrolled as controls. The study protocol was approved by the Ethics Committee of the First Affiliated Hospital of Zhengzhou University. Written informed consent was obtained from each participant.

\section{Genetic analysis}

Genomic DNA was extracted from the peripheral blood leukocytes. After PCR amplification of relevant DNA fragments from purified genomic DNA samples, the coding region and exon-intron boundaries of PSENI, $P S E N 2$ and APP (exons 16, 17) were subjected to Sanger sequencing. Alignment and analysis were carried out using the DNAStar software (DNAStar, Inc., Madison, WI). The primers were designed as previously reported [17-19]. Novel coding sequence variants detected in the patients were tested against the ethnically matched healthy controls. The alignment was also performed using UniProt ( $w w w$.uniprot.org/uniprot/). In addition, variants identified in the study were also searched in the genome reference databases, including 1000Genomes (www. internationalgenome.org/) and Exome Aggregation Consortium (ExAC, http://exac.broadinstitute.org) databases. For sporadic EOAD cases, a group of six microsatellites markers, including D14S268, D14S620, D14S1028, D14S77, D14S1004, and D14S1025, was analyzed in the family to check parenthood.

\section{Bioinformatics analysis}

Five different bioinformatics analyses based on evolutionary, structural, and computational methods were carried out to predict mutations in silico. Online bioinformatics programs were employed to evaluate the biological impact of the missense mutation in silico: sProtein Variation Effect Analyzer (PROVEAN, http//provean.jcvi.org/index.php) [20], MutPred (http://mutpred.mutdb.org/) [21], SNAP (www.rostlab. org/services/SNAP/) [22], PolyPhen2 (http://genetics. bwh.harvard.edu/pph2/) [23], Mutation Taster (www.mutationtaster.org) [24] and MutationAssessor (http://mutationassessor.org/) [25]. Homology modeling of the PSEN1 protein was also performed. The tertiary structure of the protein was downloaded from the SWISS-MODEL Repository (http://swissmodel.expasy. org/repository), and the effect of the mutations was evaluated in DeepView/Swiss-PdbViewer (version 4.0.4, http://spdbv.vitalit.ch/) [26].

Table1. The clinical characteristics of the proband in the three EOAD families.

\begin{tabular}{|c|c|c|c|}
\hline Clinical characteristics & $\begin{array}{c}\text { Proband in family } 1 \\
\text { (III-3) }\end{array}$ & $\begin{array}{c}\text { Proband in family } 2 \\
\text { (III-4) }\end{array}$ & $\begin{array}{c}\text { Proband in family } 3 \\
\text { (II -2) }\end{array}$ \\
\hline Gender & Female & Female & Female \\
\hline Family history & + & + & - \\
\hline Age onset (years) & 40 & 41 & 30 \\
\hline Disease process (years) & 5 & 1 & 4 \\
\hline Symptoms onset & Memory decline & Memory decline & Memory decline \\
\hline Psychiatric symptoms & + & + & + \\
\hline Epilepsy & + & + & + \\
\hline Extrapyramidal symptoms & + & - & - \\
\hline MMSE & 12 & 17 & 23 \\
\hline MoCA & 10 & - & 19 \\
\hline CDR & 2 & 1 & 1 \\
\hline ADAS-cog & NA & NA & 23 \\
\hline Cranial MRI & $\begin{array}{l}\text { Cerebral cortex and bilateral } \\
\text { hippocampal atrophy }\end{array}$ & $\begin{array}{l}\text { Bilateral frontoparietal lobe } \\
\text { demyelination }\end{array}$ & $\begin{array}{l}\text { Cerebral cortex and bilateral } \\
\text { hippocampal atrophy }\end{array}$ \\
\hline
\end{tabular}

MMSE: Mini-Mental State Examination; MoCA: Montreal Cognitive Assessment); CDR: Clinical Dementia Rating; ADAS-cog: Alzheimer's Disease Assessment Scale-cognitive; NA: not available 
Table 2 Bioinformatic analyses of PSEN1 variants.

\begin{tabular}{llll}
\hline Pedigrees & Family 1 & Family 2 & Family3 \\
\hline Nucleotide Change & c.766T $>$ A & c.641A $>\mathrm{G}$ & c.641A $>\mathrm{G}$ \\
Amino Acid Change & p. Y256N & P. H214R & P. G206V \\
Location & Exon7, TM-VI & Exon7, HL-IV & Exon7, TM-IV \\
ExAC & 0 & 0 & 0 \\
1000Genome & 0 & 0 & 0 \\
PROVEAN Scores & -7.2 (Deleterious) & -7.6 (Deleterious) & -8.5 (Deleterious) \\
MutPred2 scores & 0.953 (Pathogenicity) & 0.934 (Pathogenicity) & 0.926 (Pathogenicity) \\
SNAPscores & 92 (functional effect) & 85 ((functional effect) & 75 (functional effect) \\
Polyphen 2 scores (HumDiv) & 1.000 (Probably damaging) & 1.000 (Probably damaging) & 1.000 (Probably damaging) \\
Polyphen 2 scores (HumVar) & 0.999 (Probably damaging) & 0.999 (Probably damaging) & 1.000 (Probably damaging) \\
MutationTaster & Disease causing & Disease causing & Disease causing \\
MutationAssessor & High deleterious & Medium deleterious & Medium deleterious \\
\hline
\end{tabular}

\section{RESULTS}

\section{Clinical findings and genetic analysis}

The clinical characteristics of probands in the three EOAD families are shown in Table1. Sanger sequencing detected three mutations in exon 7 of the PSEN1 gene in the three families. Two novel mutations of PSEN1 were identified in families 1 and 2, respectively. Additionally, a de novo mutation was found in a $\mathrm{VEOAD}$ case in family 3.

Family 1 (PSENI Y256N)

The proband (Fig. 1A, III-3) was a 45-year-old righthanded woman. She presented with slowness in response, short-term memory impairment at 40 years of age, and progressive deterioration combined with disorientation, confusion, and cognitive disorders (mental, calculative, and comprehensive disabilities). She got the diagnosis of EOAD at age 41 years (MMSE score $=12$, MoCA score $=10, \mathrm{CDR}$ score $=2$ ). Cranial magnetic resonance imaging (MRI) uncovered bilateral temporal lobe and hippocampal atrophy (Fig. 2A). She has also had static tremor, kinetic tremor, hypermyotonia, speech disorder, and generalized tonic-clonic seizures for 42 years of age.

The proband's mother (II-2) presented with a severe memory decline and died at 45 years of age. Her older brother (III-1) had a memory decline and mental disorders for 7 years and died at age 47 years of age. Her 39-yearold sister (III-4) and another 38-year-old sister (III-5) also had memory impairment for 3 and 5 years, respectively.

Genetic analysis revealed a synonymous mutation and an adjacent nonsynonymous mutation in the PSEN1 gene in the proband, leading to a silent mutation, V255V, and a missense tyrosine-to-asparagine change, Y256N (Fig. 1B). Besides, other EOAD-affected family members (III-4, III-5) had the same mutation, but it was absent in unaffected relatives (II-1, III-2).

Family 2 (PSEN1 H214R)

The proband (Fig. 1C, III-4) was a 42-year-old, righthanded woman. She presented with memory decline, and mood and behavioral disorders at 41 years of age. The MMSE score was 17, and the CDR score was 2. Cranial MRI showed mild white matter demyelination of the frontal and parietal lobes but no obvious cerebral cortex or hippocampal atrophy (Fig. 2B).

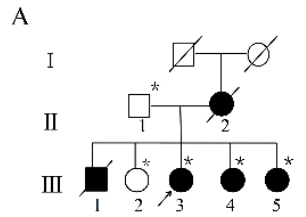

C
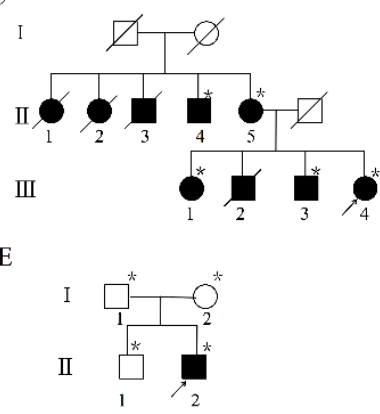

B

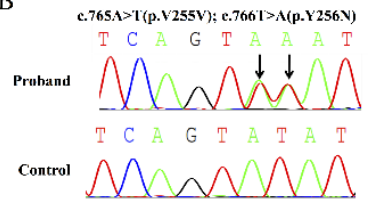

$\mathrm{D}$

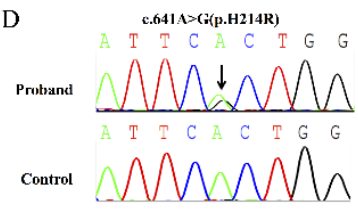

c.617.G $>\mathrm{T}(\mathrm{p} . \mathrm{G} 206 \mathrm{~V})$

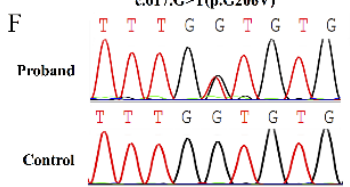

Figure 1. The pedigree charts and Sanger sequence chromatograms of the three families. A) The pedigree chart of family 1 . B) The Sanger sequence chromatogram of family 1. C) The pedigree chart of family 2. D) The Sanger sequence chromatogram of family 2. E) The pedigree chart of family 3. F) The Sanger sequence chromatogram of family 3. Open symbols, unaffected; filled symbols, affected; arrow, proband. Asterisk, members sequenced in the study. Vertical arrows indicate the mutation site.

The family history revealed that proband's three siblings (III-1, III-2 and III-3), her mother (II-5), and her mother's siblings (II-1, II-2, II-3, and II-4) were also affected by the same symptoms at onset of age at $\sim 40$ years of age. Her older sister (III-1) and older brother (III-2) had the age onset of 40 years old and died 10 years later. Her 
44-year-old brother (III-3) has had a slight memory decline for 41 years of age.

A heterozygous nucleotide substitution in exon 7 of the PSEN1 gene, c.642A $>\mathrm{G}$ (p. His214Arg), was identified in the proband (III-4; Figure 1D). This mutation was also found in his mother, his older brother, and his uncle (II-5, III-3, and II-4).

Family 3 (PSEN1 G206V)

The proband (Fig. 1E, II-2) was a 34-year-old righthanded male, who developed slowly progressing memory loss combined with irritation and anxiety at 30 years of age. His MMSE, MoCA, and CDR scores were 23, 19, and 1, respectively. The cranial MRI showed bilateral temporal lobe and hippocampal atrophy (Fig. 2C). His parents were nonconsanguineous, and none of his family members had similar symptoms.

DNA sequencing analysis revealed a heterozygous mutation (c.617G > T, p.Gly206Val) in PSEN1 in the proband (Fig. 1F), but no additional AD-affected family member was found. DNA microsatellite markers analysis indicated that the mutation had occurred de novo.
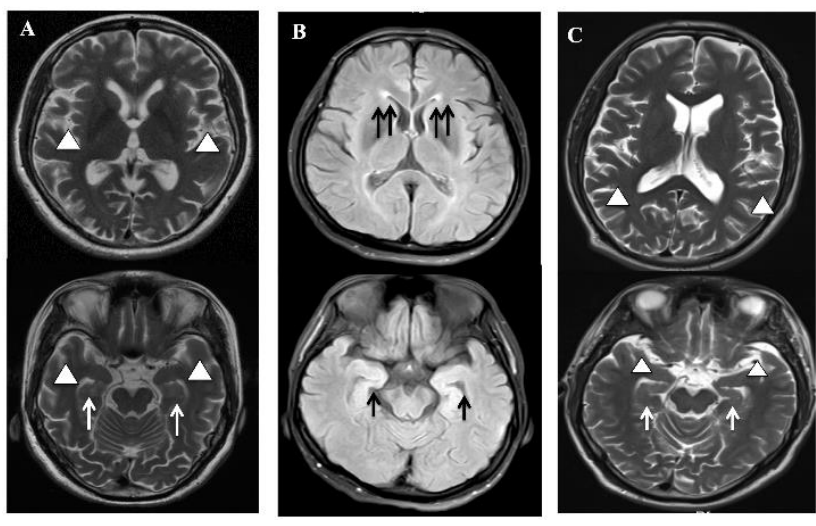

Figure 2. The cranial MRI of the probands in the three families. A) The T2 weighed image showed atrophy of cerebral cortex (arrowhead) and hippocampus (arrow) in proband (III-1) in family 1. B) The T2 FLAIR image showed mild white matter demyelination (double arrows) in frontoparietal lobe of the proband (III-2) in family 2. C) The T2 weighed MRI image showed atrophy of cerebral cortex (arrowhead) and hippocampus (arrow) in proband (III-3) of family 3.

\section{In silico predictions}

The bioinformatics analysis tools predicted that all the mutations were deleterious (Table 2). All the three mutated residues were found to be highly conserved across different species (Figure 3A). Furthermore, all the three mutations were absent in the 200 ethnically matched healthy controls and have not been reported in the dbSNP database or 1000 Genomes Project database. The tertiary structure of protein PSEN1 with wild-type residues or mutations was generated. Mutations Y256N, H214R, and
G206V resulted in changes at positions 256, 214, and 206 of the amino acid side chains of the PSEN1 protein (Fig. 3B).

\section{DISCUSSION}

In this study, we analyzed three Chinese EOAD families . Three mutations in exon 7 of the PSENI gene were identified, including two novel mutations (Y256N and $\mathrm{H} 214 \mathrm{R}$ ) in two EOAD families, and a de novo mutation $(\mathrm{G} 206 \mathrm{~V})$ in an unrelated family in a patient with vEOAD. Exon 7 of PSEN1 is one of the most frequently mutated loci, and mutations in this exon have often been found in association with vEOAD [27].

A novel mutation of PSEN1 (Y256N) was identified in this study, which is a second case with a mutation at this position. Previously, Y256S in PSEN1 has been reported to be associated with one of the youngest ages of $\mathrm{AD}$ onset (25 years old). The patient presented with aggressive $\mathrm{VEOAD}$ with severe involvement of the primary motor cortex [28]. In contrast, the vEOADaffected patients with the Y256N mutation in our study had milder clinical symptoms than did the patients with the Y256S mutation, and a relative older age at onset (40 years old). The $\mathrm{Y} 256 \mathrm{~N}$ mutation may be less harmful than the previously reported Y256S mutation, and more studies are needed to further test this hypothesis.

The second novel mutation C.641A $>\mathrm{G}(\mathrm{H} 214 \mathrm{R})$ in PSEN1 was identified in family 2 . Two known mutations have been reported at this amino acid positon. The $\mathrm{H} 214 \mathrm{Y}$ mutation was detected in two families with the age of onset ranging from 37 to 51 years old, with the varied clinical phenotypes in the same family [29, 30]. Another mutation, H214D, was also detected in one EOAD family, in which the proband developed $\mathrm{AD}$ symptoms at the age of 55 years [31]. Although the underlying pathogenesis remains unclear, the $\mathrm{H} 214 \mathrm{R}$ mutation may not be as harmful as other mutations that caused earlier onset of $\mathrm{AD}$ and typical cerebral cortex atrophy.

A de novo mutation in PSEN1, G206V, was identified in a vEOAD case in family 3 . The mutation has been reported previously in one autosomal dominant EOAD family with four affected patients in three generations. They developed memory loss in their late $20 \mathrm{~s}$ to early 30s and died in their early 40s [32]. This is the second report on this mutation to date, and we proved the previously published hypothesis that G206V in PSEN1 is a probable pathogenic mutation. Besides, other mutations (G206S, G206A, and G206D) at this amino acid position have been also reported, suggesting a mutation hotspot at this locus [33-35]. One of the largest cohort studies has identified nine de novo mutations in the PSENI gene in 129 sporadic cases with onset below age 51 [9]. This evidence also suggests that genetic analysis should be 
performed not only in familial EOAD, but also in sporadic EOAD.

Emerging research projects in Japan, Korea, and China are evaluating the genotype and clinical phenotype of $\mathrm{AD}$ [14]. According to the Alzheimer's Research Forum database (www.alzgene.org), 253 variants have been discovered in PSENI around the world. Among them, 58 variants have been discovered in Japan, Korea, China, or Malaysia. Because the aging population in most Asian countries is increasing, determining the initial pathological change is a key issue for $\mathrm{AD}$ research and treatment. Genetic analysis helps with AD diagnosis, evaluation of the pathogenesis, and finding suitable therapeutic targets in $\mathrm{AD}$ [15].

In conclusion, we reported two novel mutations in PSEN1 (Y256N and H214R) and a de novo mutation (PSEN1 G206V) detected in three Chinese EOAD families. Nevertheless, further functional studies on the three mutations are needed to determine their roles in the pathogenesis of $\mathrm{AD}$.
A

HUMAN
MOUSE
RAT
BOVIN
CHICK
DANRE
CANLF
MICMU
MACFA
PONAB
XENLA

B

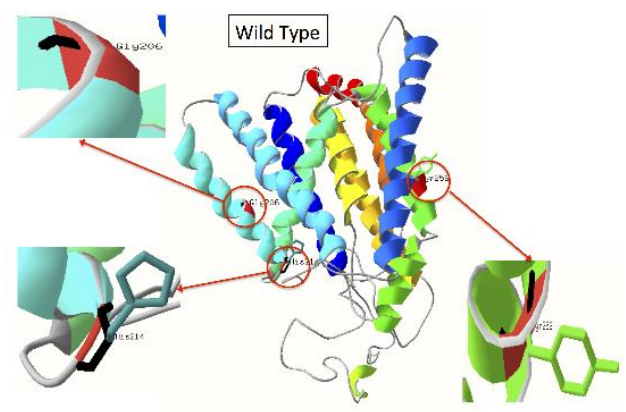

p.G206 P.H214

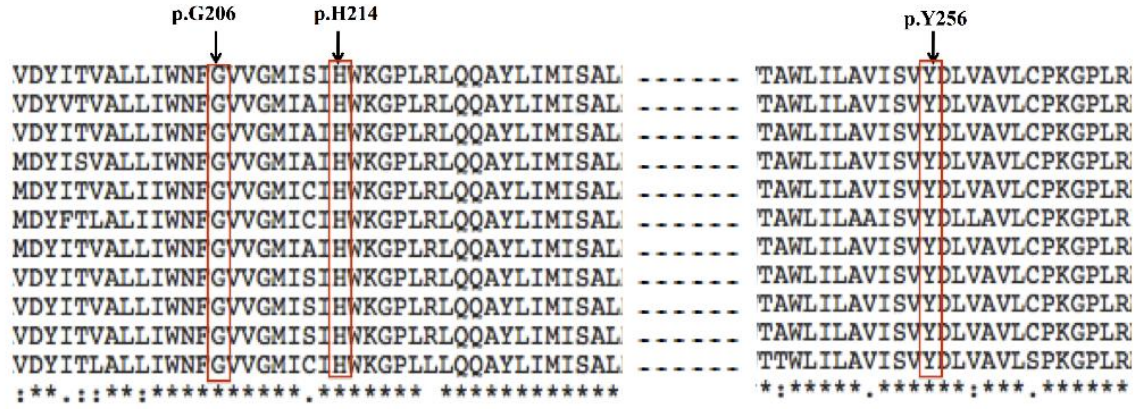

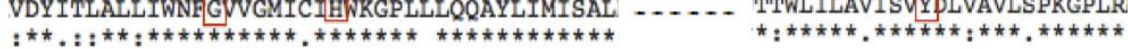

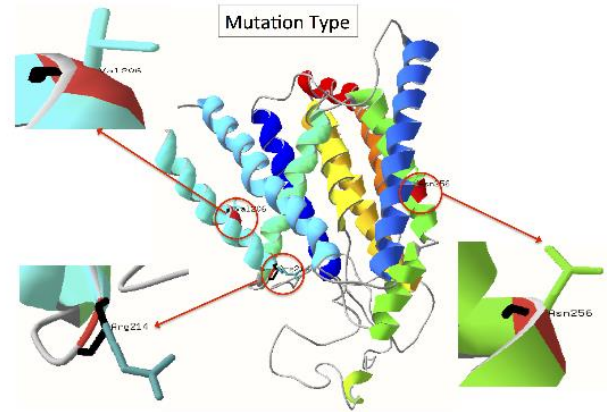

Figure 3. The conservation analysis of the three mutation sites and protein structure modeling of PSEN1. A) conservation analy sis of the three mutation sites. Arrow indicates the mutation sites. B) the 3D protein structure modeling wild type and mutation type of PSEN1 protein. The residues in codons 206, 214, and 256 were highlighted. Mutations of Y256N, H214R, and G206V change the residue side chains at the positions 256, 214, and 206 of PSEN1 protein.

\section{Acknowledgements}

This work was supported by National Key R\&D Program of China (grant 2017YFA0105000), the National Natural Science Foundation of China to Chang-he Shi (grant 81771290), the National Natural Science Foundation of China to Yu-ming Xu (grant 81530037) and Henan Foundation and Frontier technology research to Jing Yang (grant 162300410129).

\section{Conflict of interest}

The authors declare no competing financial interests.

\section{References}

[1] Robinson M, Lee BY, Hanes FT (2018). Recent Progress in Alzheimer's Disease Research, Part 2: Genetics and Epidemiology. JAlzheimers Dis, 61:459.

[2] KoedamEL, Lauffer V, van der Vlies AE, van der Flier WM, Scheltens P, Pijnenburg YA (2010). Early-versus late-onset Alzheimer's disease: more than age alone. J Alzheimers Dis, 19:1401-1408.

[3] Bagyinszky E, Youn YC, An SS, Kim S (2014). The genetics of Alzheimer's disease. Clin Interv Aging, 9:535-551.

[4] Cacace R, Sleegers K, Van Broeckhoven C (2016). Moleculargenetics of early-onset Alzheimer's disease revisited. Alzheimers Dement, 12:733-748. 
[5] Lou F, Luo X, Li M, Ren Y, He Z (2017). Very earlyonset sporadic Alzheimer's disease with a de novo mutation in the PSEN1 gene. Neurobiol Aging, 53:193 e191-193 e195.

[6] Golan MP, Styczynska M, Jozwiak K, Walecki J, Maruszak A, Pniewski J, et al. (2007). Early-onset Alzheimer's disease with a de novo mutation in the presenilin 1 gene. Exp Neurol, 208:264-268.

[7] Portet F, Dauvilliers Y, Campion D, Raux G, Hauw JJ, Lyon-Caen O, et al. (2003). Very early onset AD with a de novo mutation in the presenilin 1 gene (Met 233 Leu). Neurology, 61:1136-1137.

[8] Dumanchin C, Brice A, Campion D, Hannequin D, Martin C, Moreau V, et al. (1998). De novo presenilin 1 mutations are rare in clinically sporadic, early onset Alzheimer's disease cases. French Alzheimer's Disease Study Group. J Med Genet, 35:672-673.

[9] Lanois elee HM, Nicolas G, Wallon D, Rovelet-Lecrux A, Lacour M, Rousseau S, et al. (2017). APP, PSEN1, and PSEN2 mutations in early-onset Alzheimer disease: A genetic screening study of familial and sporadic cases. PLoS Med, 14:e1002270.

[10] Rovelet-LecruxA, Charbonnier C, Wallon D, Nicolas G, Seaman MN, Pottier C, et al. (2015). De novo deleterious genetic variations target a biological network centered on Abeta peptide in early-onset Alzheimer disease. Mol Psychiatry, 20:1046-1056.

[11] Spasic D, Tolia A, Dillen K, Baert V, De Strooper B, Vrijens S, et al. (2006). Presenilin-1 maintains a ninetransmembrane topology throughout the secretory pathway. J Biol Chem, 281:26569-26577.

[12] Gertsik N, Chiu D, Li YM (2014). Complex regulation of gamma-secretase: from obligatory to modulatory subunits. Front Aging Neurosci, 6:342.

[13] Chavez-Gutierrez L, Bammens L, Benilova I, Vandersteen A, Benurwar M, Borgers M, et al. (2012). The mechanism of gamma-Secretase dysfunction in familial Alzheimer disease. EMBO J, 31:2261-2274.

[14] Bagyinszky E, Youn YC, An SS, Kim S (2016). Mutations, associated with early-onset Alzheimer's disease, discovered in Asian countries. Clin Interv Aging, 11:1467-1488.

[15] Xu Y, Liu X, Shen J, Tian W, Fang R, Li B, et al. (2018). The Whole Exome Sequencing Clarifies the Genotype- Phenotype Correlations in Patients with Early-Onset Dementia. Aging Dis, 9:696-705.

[16] Dubois B, Feldman HH, Jacova C, Dekosky ST, Barberger-Gateau P, Cummings J, et al. (2007). Research criteria for the diagnosis of Alzheimer's disease: revising the NINCDS-ADRDA criteria. Lancet Neurol, 6:734-746.

[17] Cruts M, van Duijn CM, Backhovens H, Van den Broeck M, Wehnert A, Serneels S, et al. (1998). Estimation of the genetic contribution of presenilin-1 and -2 mutations in a population-based study of presenile Alzheimer disease. HumMolGenet, 7:43-51.

[18] Schellenberg GD, Pericak-Vance MA, Wijsman EM, Moore DK, Gaskell PC, Jr., Yamaoka LA, et al. (1991). Linkage analys is of familial Alzheimer disease, using chromosome 21 markers. Am J Hum Genet, 48:563-
583.

[19] Tanzi RE, Vaula G, Romano DM, Mortilla M, Huang TL, Tupler RG, et al. (1992). Assessment of amyloid beta-protein precursor gene mutations in a large set of familial and sporadic Alzheimer disease cases. Am J Hum Genet, 51:273-282.

[20] Choi Y, Sims GE, Murphy S, Miller JR, Chan AP (2012). Predicting the functional effect of amino acid substitutions and indels. PLoS One, 7:e46688.

[21] Pejaver V, Urresti J, Lugo-Martinez J, Pagel KA, Lin GN, Nam H-J, et al. (2017). MutPred2: inferring the molecular and phenotypic impact of amino acid variants. bioRxiv.

[22] Bromberg Y, Yachdav G, Rost B (2008). SNAP predicts effect of mutations on protein function. Bioinformatics, 24:2397-2398.

[23] Adzhubei IA, Schmidt S, Peshkin L, Ramensky VE, Gerasimova A, Bork P, et al. (2010). A method and server for predicting damaging missense mutations. Nat Methods, 7:248-249.

[24] Schwarz JM, Cooper DN, Schuelke M, Seelow D (2014). MutationTaster2: mutation prediction for the deep-sequencing age. Nat Methods, 11:361-362.

[25] Reva B, Antipin Y, Sander C (2011). Predicting the functional impact of protein mutations: application to cancer genomics. Nucleic Acids Res, 39:e118.

[26] Waterhouse A, Bertoni M, Bienert S, Studer G, Tauriello G, Gumienny R, et al. (2018). SWISSMODEL: homology modelling of protein structures and complexes. Nucleic Acids Res, 46:W296-W303.

[27] Bird TD. 1993. Early-Onset Familial Alzheimer Disease. In GeneReviews (R)). M.P. Adam, H.H. Ardinger, R.A. Pagon, S.E. Wallace, L.J.H. Bean, K. Stephens, and A. Amemiya, editors. Seattle WA: University of Washington, Seattle. GeneReviews is a regis tered trademark of the University of Was hington, Seattle.

[28] Miklossy J, Taddei K, Suva D, Verdile G, Fonte J, Fisher C, et al. (2003). Two novel presenilin-1 mutations (Y256S and Q222H) are associated with early-onset Alzheimer's disease. Neurobiol Aging, 24:655-662.

[29] Raux G, Guyant-Marechal L, Martin C, Bou J, Penet C, Brice A, et al. (2005). Molecular diagnosis of autosomal dominant early onset Alzheimer's disease: an update. J Med Genet, 42:793-795.

[30] Lohmann E, Guerreiro RJ, Erginel-Unaltuna N, Gurunlian N, Bilgic B, Gurvit H, et al. (2012). Identification of PSEN1 and PSEN2 gene mutations and variants in Turkish dementia patients. Neurobiol Aging, 33:1850 e1817-1827.

[31] Guerreiro RJ, Baquero M, Blesa R, Boada M, Bras JM, Bullido MJ, et al. (2010). Genetic screening of Alzheimer's disease genes in Iberian and African samples yields novel mutations in presenilins and APP. Neurobiol Aging, 31:725-731.

[32] Goldman JS, Reed B, Gearhart R, Kramer JH, Miller BL (2002). Very early-onset familial Alzheimer's disease: a novel presenilin 1 mutation. Int J Geriatr Psychiatry, 17:649-651. 
[33] Arnold SE, Vega IE, Karlawish JH, Wolk DA, Nunez $\mathrm{J}$, Negron M, et al. (2013). Frequency and clinicopathological characteristics of presenilin 1 Gly206Ala mutation in Puerto Rican Hispanics with dementia. J Alzheimers Dis, 33:1089-1095.

[34] Dobricic V, Stefanova E, Jankovic M, Gurunlian N, Novakovic I, Hardy J, et al. (2012). Genetic testing in familial and young-onset Alzheimer's disease: mutation spectrum in a Serbian cohort. Neurobiol Aging, 33:1481 e1487-1412.

[35] Park HK, Na DL, Lee JH, Kim JW, Ki CS (2008). Identification of PSEN1 and APP gene mutations in Korean patients with early-onset Alzheimer's disease. J Korean Med Sci, 23:213-217. 